\title{
Computer Programs for Performing Hierarchical Cluster Analysis
}

\author{
Mark S. Aldenderfer \\ University of Missouri-St. Louis
}

\author{
Roger K. Blashfield \\ University of Florida
}

This paper analyzes the versatility of 10 different popular programs which contain hierarchical methods of cluster analysis. The intent of the paper is to provide users with information which can be of assistance when choosing a cluster analysis program. The four dimensions which are emphasized when discussing these programs are (1) agglomeration vs. division, (2) linkage form, (3) similarity measure, and (4) hierarchical solution vs. singlerank.

Corresponding with the rapid growth of interest in cluster analysis as a method for forming objective classifications has been the development of a large number of computer programs and packages which have made the technique widely available. Blashfield (1976a) has documented that well over fifty different programs and packages which perform cluster analysis are currently in use. This figure probably underestimates the amount of computer software developed to perform these techniques, and more realistic estimates range into the hundreds.

A consumer-oriented evaluation of this large amount of software is clearly required because there is a striking diversity in the quality, versatility, and usability of these programs. Some programs are user-oriented, whereas others pro-

APPLIED PSYCHOLOGICAL MEASUREMENT

Vol. 2. No. 3 Summer 1978 pp. 403-411

(c) Copyright 1978 West Publishing Co. vide the user with only the barest information on the clustering process and its solution. All programs differ in terms of the number and types of linkage options available, data handling features, similarity options, graphics, and cluster diagnostics. This report is intended to provide users with information that will help them choose the programs most appropriate for their needs and also inform them of the advantages and disadvantages of popular programs.

Because of the enormous literature on hierarchical methods of cluster analysis (Sneath \& Sokal, 1973), it is impossible for any single researcher to attempt a truly comprehensive review. Also, because the number of programs developed to implement these methods has grown rapidly in the last 13 years, only the most popular and commonly available programs will be reviewed. There are three general sources of clustering programs: (1) statistical "packages," (2) programs which perform only one type of cluster analysis, and (3) programs collected in books. The statistical packages discussed in this paper are BMP, CLUSTAN, NTPAK, NTSYS, OSIRIS, and SAS. The programs emphasizing only one method of clustering analysis are HGROUP and HICLUS. Finally, books by Anderberg (1973), denoted as ANDER, and Hartigan (1975), denoted as HART, are included. These 10 programs are among the 14 most popular, as determined through a ques- 
tionnaire sent to cluster analysis users (Blashfield, 1976a).

The primary emphasis of this report is an assessment of the versatility of clustering software. Versatility is defined as the range of hierarchical options available to the user, the clarity and completeness of interpretive aids, and the flexibility of the software for application to a wide variety of research problems. Versatility is especially important when considering cluster analysis because of the confusion surrounding the evaluation of the adequacy of clustering results. Because no consensus on appropriate evaluative criteria exists, it is essential that the user be aware of the many types of cluster analysis and the specific properties of each method. Moreover, no single clustering method is applicable to all research problems; the option to choose the most suitable method for a particular research problem is thus extremely important.

Specifically, this report will focus on the general hierarchical options and graphics available in each program or package. Interested readers will find more detailed discussions of other types of clustering software in a series of unpublished "Consumer Reports" available from the second author (Blashfield \& Aldenderfer, 1977; Aldenderfer, 1977; Blashfield, 1977a, 1977b). ${ }^{1}$

\section{General Hierarchical Options}

There have been numerous attempts to specify the most important dimensions of variability said to characterize all clustering methods. Bailey (1974) has listed 12 "criteria" to be used in choosing a clustering method; Sneath and Sokal (1973) have presented 8 "options" which also serve to guide choice of method. Four of these dimensions are crucial for the understanding of hierarchical clustering methods: (1) agglomeration vs. division, (2) linkage form, (3) similarity measure, and (4) hierarchical solution

\footnotetext{
"Copies of the four "Consumer Reports" on cluster analysis software can be obtained for one dollar $(\$ 1.00)$ from the address given at the end of this article.
}

vs. single-rank solution. Table 1 summarizes the characteristics of the 10 programs along these 4 dimensions.

\section{Agglomeration vs. Division}

In hierarchical clustering systems, there are two basic strategies of creating a classification: agglomeration or division. Agglomerative methods begin with $N$ individuals (cases, operational taxonomic units, or OTUs), each in its own cluster, and then combine individuals into more clusters until only one cluster exists. That is, if $K$ is defined as the number of clusters, $K=N$ at the start of the procedure; and at the end, $K=1$. Divisive procedures are the exact opposite: $K=1$ at the start of a divisive procedure, and $K=N$ at its termination (or the endpoint value of $K$ in divisive methods can be determined by the user). Of the 10 programs, only CLUSTAN contains a divisive method; all 10 programs contain agglomerative methods.

\section{Linkage Form}

Linkage form refers to the set of rules used in agglomerative methods to join individuals together to create groups or clusters. Linkage types have been described by many authors in great detail. Sneath and Sokal (1973) provide an exceptionally clear representation of how each of the linkage types works by using hypothetical data sets to illustrate the sequence of links which create the dendrogram. Everitt (1974) also provides simple examples of linkage form differences. The following are the most common linkage types found in hierarchical agglomerative clustering:

Single linkage clustering. First discussed by Sneath (1977), this method of clustering specifies that an individual may join a cluster if it has a certain level of similarity with at least one of the members of that cluster. Connections between entities and clusters and between two clusters are thus based on links between single entities. In application, single linkage leads to 
Table 1

Characteristics of Popular Software Programs for Hierarchical Cluster Analysis

\begin{tabular}{|c|c|c|c|c|}
\hline Program & $\begin{array}{c}\text { Agglomeration } \\
\text { vs. } \\
\text { Division }\end{array}$ & $\begin{array}{c}\begin{array}{c}\text { Number } \\
\text { of } \\
\text { Linkage Forms }\end{array} \\
\end{array}$ & $\begin{array}{c}\text { Number } \\
\text { of } \\
\text { Similarity } \\
\text { Measures }\end{array}$ & $\begin{array}{c}\text { Hierarchical } \\
\text { Solution } \\
\text { vs. } \\
\text { Single Rank }\end{array}$ \\
\hline ANDER & Agglomerative & 7 & 15 & Both \\
\hline BMDP & Agglomerative & 3 & 9 & Both \\
\hline CLUSTAN & Both & 10 & 38 & Both \\
\hline HART & Agglomerative & 2 & 4 & Hierarchical \\
\hline HGROUP & Agglomerative & 1 & 1 & Both \\
\hline HICLUS & Agglomerative & 2 & none & Hierarchical \\
\hline NTPAK & Agglomerative & 3 & 4 & Hierarchica1 \\
\hline NTSYS & Agglomerative & 6 & 21 & Hierarchical \\
\hline OSIRIS & Agglomerative & 1 & none & Hierarchical \\
\hline SAS & Agglomerative & 1 & 1 & Both \\
\hline
\end{tabular}

the formation of long, straggly clusters, described as chaining. Despite this difficulty, one "school" of numerical taxonomy claims that this method is to be preferred because it is the only method satisfying a set of analytical criteria which should be satisfied by all methods. (Jardine \& Sibson, 1968a, 1968b, 1971). All programs except HGROUP, OSIRIS, and SAS contain the single linkage method as an option.

Complete-linkage clustering. This method is logically the opposite of single-linkage analysis. Instead of an entity joining with only one entity in a cluster, complete linkage requires that an entity be within a specified level of similarity with all members of that cluster. This method tends to form compact, hyperspherical clusters which join one another only at very low levels of similarity. Only HART and HGROUP do not contain a complete linkage option.

Average linkage methods. These were first discussed by Sokal and Michener (1958) as an antidote to the extremes of single and complete linkage. Essentially, these methods compute an average of the similarity of an entity with the entities of a cluster and will join the entity to the cluster if a given level of similarity is achieved using this average. A number of definitions of computing the average similarity exist. One of these is the arithmetic average, in which the average of the similarity values between entities and cluster are computed. Sneath and Sokal (1973) differentiate the use of arithmetic averaging from centroid clustering. In the latter method, the first step is to calculate the centroid of each cluster and then to measure the similarity between that centroid and the centroid of the cluster or entity which joins it. Average linkage methods can be further specified by noting if weighting of the clusters is permitted. There has been a long debate in biology concerning whether or not a researcher should a priori weight clusters joining another on the basis of the number of entities each may possess. (The interested reader may consult Sokal \& Michener, 1958, and Sneath, 1969, for details.) The four prominent methods of average linkage cluster analysis are

1. Unweighted pair-group method using arithmetic averages (UPGMA; available in ANDER, BMDP, CLUSTAN, HARTIGAN, NTPAK, and NTSYS) 
2. Weighted pair-group method using arithmetic averages (WPGMA; available in NTSYS and CLUSTAN)

3. Unweighted pair-group centroid method (UPGMC; available in NTPAK, NTSYS, and CLUSTAN)

4. Weighted pair-group centroid method (WPGMC; available in NTSYS and CLUSTAN)

UPGMA is by far the most commonly used of these methods and is the only one of these methods to be recommended if group averages are sought (see Sneath \& Sokal, 1973, for relevant arguments).

Minimum variance methods. While a number of these methods exist, the best known is Ward's (1963) method. This method erects a hierarchical tree by satisfying an objective function each time the program joins entities and/or clusters. In Ward's method, the objective function is the sum of the within-group sum of squares, also known as the error sum of squares. At each stage in the analysis, every possible pair of combinations of clusters is considered, and the pair that produces the minimum increase in the objective function is clustered together. This method has been virtually ignored in the biological literature but has been extensively used in many of the social sciences. This method generally finds clusters of spherical shape but also has a tendency to find clusters of equal size. Monte carlo comparisons of cluster analysis methods have favored Ward's method (Blashfield, 1976b; Hubert, 1972). ANDER, CLUSTAN, and HGROUP are the only programs containing Ward's method.

CLUSTAN is by far the most versatile package in terms of the number of methods available. CLUSTAN contains 10 hierarchical methods, including 2 divisive methods. ANDER (7 methods) and NTSYS (6 methods) also show high versatility, with the only popular method absent from NTSYS being Ward's method. The BMDP procedures have moderate versatility. The BMDP1M procedure has 3 options-single, complete, and average linkage- - but this procedure only clusters variables. BMDP2M, designed to cluster cases, has one option-UPGMA. OSIRIS, HICLUS, SAS, HART, and NTPAK are the least versatile of the programs and packages under discussion from the standpoint of linkage options.

\section{Similarity Measures}

All hierarchical methods of clustering require the calculation of a similarity matrix of the cases or OTUs used in the clustering exercise. ${ }^{2}$ If the number of cases is $N$, the order of the similarity matrix is $N \times N$. That is, the similarity of each case to every other case is calculated, and these values are used by the clustering method to create clusters. Similarity can be defined as the degree of value of resemblance an entity has with another entity on the basis of the characters or attributes each of them possesses. Dissimilarity, of course, is the converse, being in some sense the "distance" between entities. High similarity values imply low dissimilarity values, and vice versa.

The literature on how to choose a similarity coefficient is almost as confused and complex as that which surrounds the choice of the sorting strategy. It has been observed repeatedly that different similarity measures used with the same clustering method can produce quite different results. The concept of similarity varies from problem to problem, and the researcher must choose coefficients on the basis of two general criteria: (1) what metrics best assess the characteristics of the entities and (2) what similarity measures best express resemblance among en-

\footnotetext{
${ }^{2} \mathrm{~A}$ note of caution on terminology and representation is appropriate. Jardine and Sibson (1971b) refer to the $N \times N$ matrix of similarities as raw data. Normally, this term is reserved for the $N \times P$ matrix of cases and their attributes, where $P$ is the number of attributes. Also, the representation of the $N \times P$ matrix differs in the biological sciences. In biology, the rows are the attributes $(P)$ and the columns are the cases $(N)$. In the nonbiological sciences, this is usually reversed.
} 
tities. As with the choice of clustering method, no single similarity measure is appropriate for all problems.

Following Sneath and Sokal (1973), the large body of similarity measures can be divided into four major groups:

1. Distance measures are generally used with continuous or ordinal data but are applicable to binary/qualitative data as well. The user must be aware that many types of distance exist, and not all of them need be similar to the Euclidean metric. (Distance metrics are discussed at length by Gower, 1972).

2. Association coefficients are generally used with binary, categorical, or qualitative data.

3. Correlation coefficients, such as Pearson's $r$, are used for continuous data.

4. Probabilistic similarity coefficients are based on information statistics. These are unique in that instead of a measure of similarity being computed between entities, the information gain of the combination of two entities is evaluated, and the pair which provides the least information gain (in the Shannon sense) is fused. This type of measure works directly from the raw data and does not require the estimation of similarity between entities in the traditional sense of the term.

The variability in the number of similarity measures among the packages is great, ranging from 1 to 38. CLUSTAN has by far the most extensive range of coefficients (38), covering all 4 coefficient types discussed above. NTSYS has 21 similarity measures, including all but a probabilistic coefficient; ANDER has 15 similarity options; and BMDP has 9. None of the remaining programs contains more than 2 similarity measures.

Two programs, HICLUS and OSIRIS, are unique in that they require the user to input the similarity matrix instead of the raw data. All programs except HGROUP will permit the user to input a similarity matrix as an option.
Finally, it should be noted that optional procedures in two programs-BMDP, using BMDP1M, and OSIRIS, using CLUSTR-permit the user to cluster variables instead of objects.

\section{Hierarchical vs. Single-Rank}

This dimension of hierarchical clustering methods refers to the nature of the relationships among cases at the finish of the clustering procedure. The most familiar representation of a hierarchical system is that of a "tree," or dendrogram, as defined by Hartigan (1967). Ranks in a tree or dendrogram are represented by the level of similarity at which individuals, groups of individuals, and finally groups of groups of individuals are successively joined. Thus, an individual may be simultaneously a member of clusters at two or more ranks; that is, clusters formed early in the clustering procedure can be members of "higher order" clusters joined at later stages.

The concept of hierarchical representation of a data set was developed primarily in biology. The structure of output from a hierarchical clustering method resembles the traditional hierarchical structure of Linnean taxonomy with its graded sequence of ranks. The preference for hierarchical clustering methods in the biological sciences is further strengthened by a "school" of taxonomic thought which claims that it is possible to reconstruct the phylogenetic relationships of organisms using numerical taxonomy. This school of thought was first expressed in Principles of Numerical Taxonomy by Sokal and Sneath (1963), which revolutionized the development of cluster analysis.

In contrast to the biological sciences, which favor classifications having a hierarchical structure, many of the social sciences are generally interested in single-rank classifications. The intent behind this use of clusters is to produce a given number of homogeneous clusters, which may or may not be related in a hierarchical fashion. Iterative partitioning methods of clustering are 
the methods used most frequently to produce clusters of single rank.

Although most trees generated by hierarchical methods are interpreted as wholes (i.e., on the nature of the linkages and levels of fusion of the entities), they can also be "cut" to form single-rank classifications. To accomplish this, the user must decide how many clusters exist in the data set and then scan the tree to find the level of similarity at which this number of clusters is produced. A line drawn at right angles to the tree at the level produces the desired number of clusters.

The most popular form of graphic output from the hierarchical cluster analysis is the dendrogram. This is the well-known "tree" which provides the user with a visual representation of the results of the cluster analysis. This visual representation is extremely helpful in the evaluation of the results of the clustering. As might be expected, the programs and packages differ considerably in their representation of the tree or dendrogram.

The output of a tree is standard for most programs; but some packages, such as NTSYS and CLUSTAN, require the user to include control cards with output specifications so the tree can be printed. All other packages have as standard default options the drawing of a tree. The exception to this is CLUSTAN. While CLUSTAN IC has an extensive graphics package, the graphics are not produced by the line printer. Separate job steps must be run in order to obtain a dendrogram from an off-line plotting device. The portability of these off-line graphic features from CLUSTAN may present difficulty at some computer centers. All other packages and programs have line printer graphics.

NTSYS generates the most easily visualized dendrograms from a line printer. An example of an NTSYS tree is shown in Figure 1. From this figure it can be seen that at the first step, Entities 1 and 5 join to form a cluster; at the next step, Entity 2 joins the clusters of 1 and 5 , while 8 and 10,3 and 4 , and 6 and 7 also merge. The dendrograms of the remaining packages are not as well organized visually. For instance, the tree from BMDP2M (Figure 2) is difficult to read because of its compactness, orientation, and the lack of easily observable "nodes" in the tree where entities and clusters fuse. A revision of BMDP2M generates a tree which is much

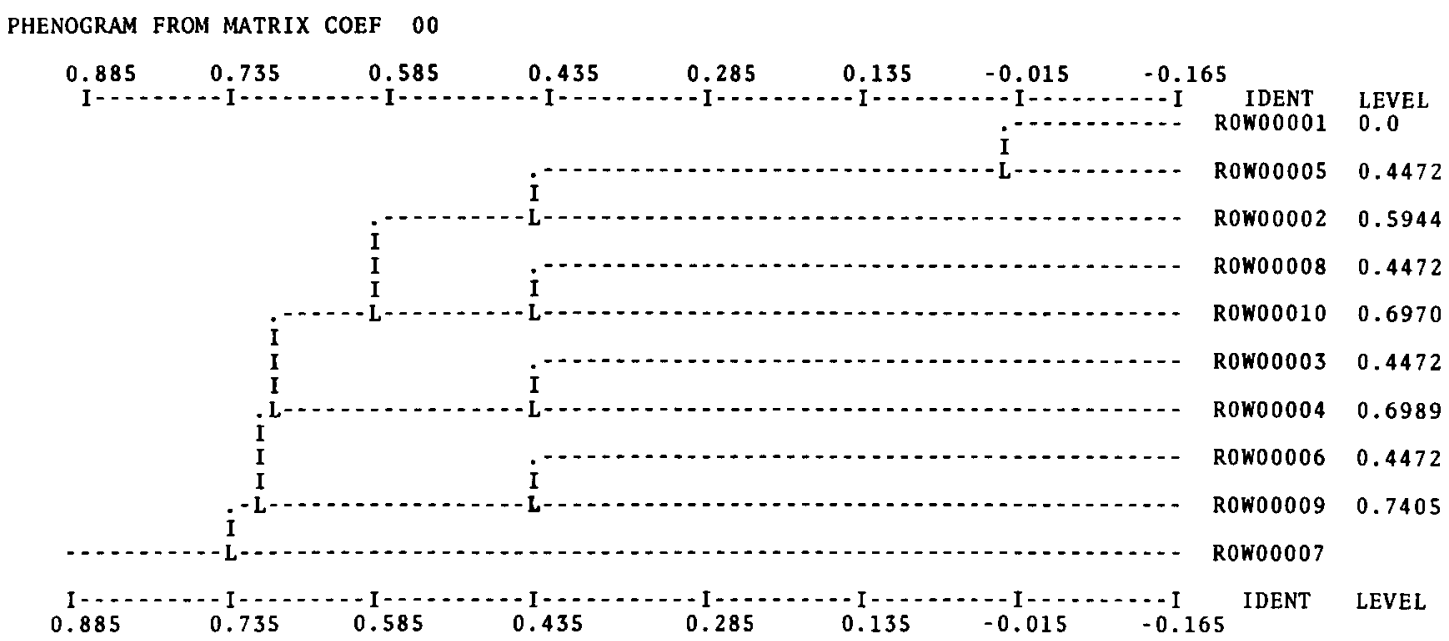


Figure 2

BMDP Tree

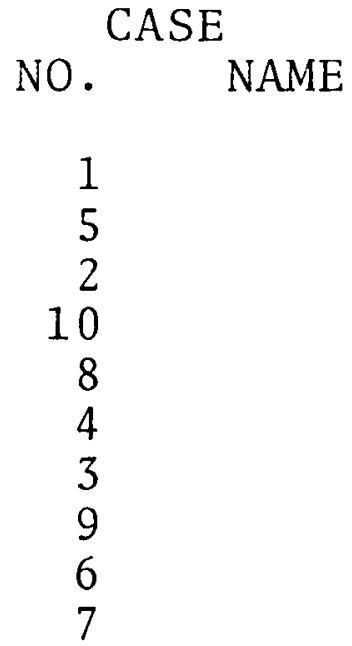

easier to interpret. The same problem exists with the trees formed by HICLUS, OSIRIS, and SAS (see example of SAS plot in Figure 3). This type of tree is better described as "sky-line plot" because of its shape. Although the nodes can be discerned, these plots do not have the visual crispness of the trees produced by NTSYS and ANDER.

CLUSTAN, SAS, HGROUP, BMDP, and ANDER are the five programs which make it relatively easy for a user to determine a singlerank solution. Both programs provide output which details the agglomeration, or division, occurring at each step in the hierarchial clustering process. In this regard, CLUSTAN's output is the most readable. CLUSTAN will output, as an option, the entire membership list of entities in each cluster for a given number of clusters (rank) and the means and standard deviations of these clusters. The latter output (membership lists and descriptive statistics) is very useful to any user interested in a single-rank solution from a hierarchical method and is only available at present in CLUSTAN.

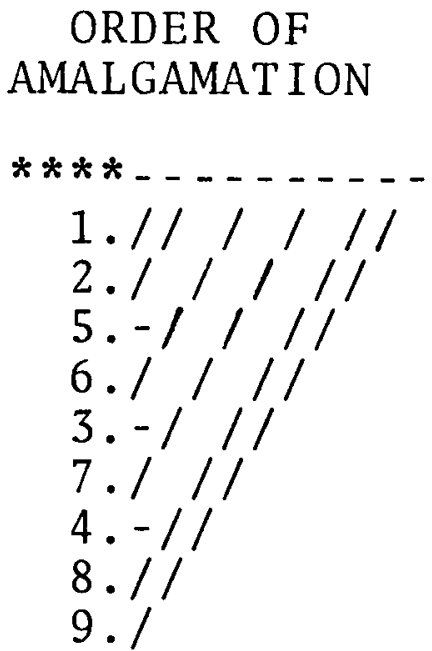

\section{Conclusion}

Of these 10 programs, CLUSTAN and NTSYS are the most versatile and flexible of the most popular existing clustering programs. Each of them contains a wide variety of clustering methods and similarity options. For the biological scientist, NTSYS is superior because of its easily generated dendrogram and other features, such as the cophenetic correlation coefficient, which are specifically designed for biological usage. Also, its user marual is couched in the jargon of numerical taxonomy, and the logic of the entire system is well suited for biological problems. CLUSTAN is the program of choice for general users, particularly those in the non-biological sciences, although biologists will also appreciate some of CLUSTAN's features. CLUSTAN will be preferred by those researchers interested in single-rank solutions because of the superior output features (other than graphics) which describe each level of clustering. If the graphics package of CLUSTAN can be made available at an institution for 
Figure 3

SAS Tree

\section{CLUSTER MAP}

\section{CASES}

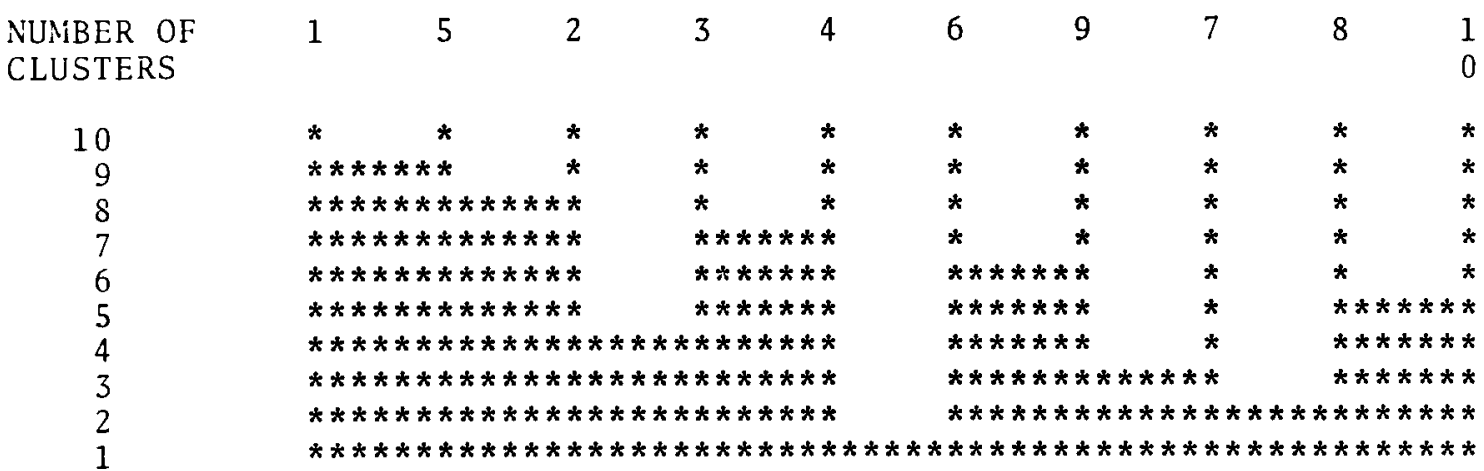

a reasonable cost, it is clearly superior to NTSYS in many areas of user interest.

Of the other packages and programs considered, only ANDER shows comparable versatility; however, the portability of ANDER is somewhat limited because the programs were written for CDC systems. Nevertheless, it was found that adapting these programs for IBM FORTRAN compliers was fairly easy. While the ANDER collection of programs offers a reasonable amount of versatility, they are not user oriented, and their use presupposes sophistication with FORTRAN and Anderberg's book.

\section{References}

Aldenderfer, M.S. A consumer report on cluster analysis software: (2) Hierarchical methods (Report No. 2, N.S.F. Grant 74-20007). July 1977.

Anderberg, M.R. Cluster analysis for applications. New York: Academic Press, 1973.

Bailey, K.D. Cluster analysis. In D. Heise (Ed.), Sociological methodology. San Francisco: JosseyBass, 1974.

Blashfield, R.K. Questionnaire on cluster analysis software. Classification Society Bulletin, 1976, 3, 25-42. (a)

Blashfield, R.K. Mixture model tests of cluster analysis: Accuracy of four agglomerative hierarchical methods. Psychological Bulletin, 1976, 83, 377-388. (b)

Blashfield, R.K. A consumer report on cluster analysis software: (3) Iterative partitioning methods (Report No. 3, N.S.F. Grant 74-20007). March 1977. (a)

Blashfield, R.K. A consumer report on cluster analy. sis software: (4) Usability (Report No. 4, N.S.F. Grant 74-20007). March 1977. (b)

Blashfield, R.K., \& Aldenderfer, M.S. A consumer report on cluster analysis software: (1) Clustering methods and their literature (Report No. 1, N.S.F. Grant 74-20007). February 1977.

Everitt, B.S. Cluster analysis. London: Halstead Press, 1974.

Gower, J.C. Measurements of taxonomic distance and their analysis. In J.S. Weiner \& J. Huzinga (Eds.), The assessment of population affinites. Oxford: Clarendon Press, 1972.

Hartigan, J.A. Representation of similarity matrices by trees. Journal of the American Statistical Association, 1967, 62, 1140-1158.

Hartigan, J.A. Clustering algorithms. New York: Wiley, 1975.

Hubert, L. Some extensions of Johnson's hierarchical and nonhierarchical classifications. Psychometrika, 1972, 37, 261-274.

Jardine, N., \& Sibson, R. Construction of hierarchical and nonhierarchical classifications. Computer Journal, 1968, 13, 116-117. (a)

Jardine, N., \& Sibson, R. A model for taxonomy. Mathematical Biosciences, 1968, 465-482. (b) 
Jardine, N., \& Sibson, R. Mathematical taxonomy. New York: Wiley, 1971.

Sneath, P.H.A The application of computers to taxonomy. Journal of General Microbiology, 1957, 17, 201-226.

Sneath, P.H.A. Evaluation of clustering methods. In A.J. Cole (Ed.), Numerical taxonomy. London: Academic Press, 1969.

Sneath, P.H.A., \& Sokal, R.R. Numerical taxonomy. San Francisco: W.H. Freeman, 1973.

Sokal, R.R., \& Michener, C.D. A statistical method for evaluating systematic relationships. Kansas University Science Bulletin, 1958, 38, 1409-1438.

Sokal, R.R., \& Sneath, P.H.A. Principles of numerical taxonomy. San Francisco: W.H. Freeman, 1963.

Ward, J.H. Hierarchical grouping to optimize an ob- jective function. Journal of the American Statistical Association, 1963, 58, 236-244.

\section{Acknowledgments}

This report was supported by a grant from the $\mathrm{Na}$ tional Science Foundation (grant DCR \#74-20007). Appreciation is extended to Lyle Schoenfeldt for his helpful suggestions on the preparation of this paper.

\section{Author's Address}

Roger K. Blashfield, Box J-256, JHMHC, Department of Psychiatry, University of Florida, Gainesville, FL 32610 